\title{
Addressing the Cold-Start Problem in Location Recommendation Using Geo-Social Correlations
}

\author{
Huiji Gao • Jiliang Tang • Huan Liu
}

Received: date / Accepted: date

\begin{abstract}
Location-based social networks (LBSNs) have attracted an increasing number of users in recent years, resulting in large amounts of geographical and social data. Such LBSN data provide an unprecedented opportunity to study the human movement from their socio-spatial behavior, in order to improve location-based applications like location recommendation. As users can check-in at new places, traditional work on location prediction that relies on mining a user's historical moving trajectories fails as it is not designed for the cold-start problem of recommending new check-ins. While previous work on LBSNs attempting to utilize a user's social connections for location recommendation observed limited help from social network information. In this work, we propose to address the cold-start location recommendation problem by capturing the correlations between social networks and geographical distance on LBSNs with a geo-social correlation model. The experimental results on a real-world LBSN dataset demonstrate that our approach properly models the geo-social correlations of a user's cold-start check-ins and significantly improves the location recommendation performance.
\end{abstract}

Keywords Location-Based Social Networks · Location Recommendation • Location Prediction · Cold-Start · Geo-Social Correlation

Huiji Gao

Arizona State University, Tempe, AZ 85287, USA

E-mail: Huiji.Gao@asu.edu

Jiliang Tang

Arizona State University, Tempe, AZ 85287, USA

E-mail: Jiliang.Tang@asu.edu

Huan Liu

Arizona State University, Tempe, AZ 85287, USA

E-mail: Huan.Liu@asu.edu 


\section{Introduction}

Location-based social media attracts millions of users, and generates large location-based social networks [20. A recent survey from the Pew Internet and American Life Project reports that over $28 \%$ of Americans use mobile or social location-based services 38. Typical online location-based social networking sites such as Foursquar 1 and Facebook Place 2 provide location-based services for users to "check-in" at a physical place, and automatically include the location into their posts. The online "check-in" posts a user's current geographical location, making known to his friends the information on when and where he is. Compared with many other online activities, "check-in" reflects a user's geographical action in the real world, residing where the online world and real world intersect. Thus, the study of check-ins provides an ideal environment to understand human behavior, and could also benefit a variety of location-based services such as mobile marketing [4, 21] and disaster relief [19, 11. Among various applications on LBSNs, location recommendation has become a significant task in recent years since it is proposed to help users filter out uninteresting items and reduce time in decision making, which could also benefit virtual marketing.

One of the most significant properties of check-in behavior is the userdriven property 27. When using location-based social networking services, a user is able to choose where and when to make a check-in. It is reported in previous research that a user's check-ins displays a power-law distribution on LBSNs, i.e., a user goes to a few places many times and to many places a few times [15], indicating that users do visit new places, resulting in the cold-start check-in problem. Recommending a none cold-start location to a user (also referred to as "location prediction") has been widely studied by taking advantage of spatial trajectories [25,32, periodical patterns [34, spatial-temporal patterns 29, 17, etc. The success of these methods relies on sufficient numbers of observations on the target location in an individual's check-in history; hence, it is difficult to apply them to the cold-start check-ins as there is no historical information on the user for the new place he will go to.

Facing the difficulty of recommending cold-start check-in locations, researchers resort to social network information on LBSNs and investigate if it could help solve the recalcitrant cold-start problem. As suggested by social theories (e.g., social correlation [1]), human movement is usually affected by their social networks, such as watching movies with families, visiting friends, traveling by following friends' recommendations, and so on, providing the potential opportunity to solve the cold-start recommendation problem from a user's social friends. However, recent work on utilizing social information for location recommendation has reported limited improvement $9,15,36,37$. One explanation of this phenomenon could be the check-in characteristics of LBSNs. It has been reported that in general users with social connections only

\footnotetext{
1 https://foursquare.com

2 https://www.facebook.com/about/location
} 
share less than $10 \%$ common check-in locations [15,9], which provide very limited observation for social recommendation.

Since the check-in action connects a user's geographical movement and his social networks, it actually provides a new perspective to study a user's cold-start check-in behavior not only through social aspect but also from the closely correlated geographical aspect, i.e., geo-social perspective. Researchers investigated how geographical distance influences social networks, and how social networks influence human movement on LBSNs 30,8,31, indicating the necessity to consider these two factors together when studying human mobile behavior, and suggesting the potential opportunity to improve current location recommendation approaches. In this paper, we propose the concept of geosocial correlations to combine both social networks and geographical distance for recommending cold-start check-in locations. In particular, we study the following issues:

- Are user's cold-start check-ins correlated to their social ties on LBSNs?

- How to capture the social correlations on LBSNs? and

- How to utilize the social correlations for solving the cold-start location recommendation problem?

To the best of our knowledge, this work presents the first comprehensive study of geo-social correlations for the cold-start problem on location-based social networks. The contributions of our work are summarized below:

- We study the usability of social network information on LBSNs, and propose a feasible solution for the cold-start location recommendation problem by taking advantage of geo-social correlations.

- We investigate the social correlations in geo-social perspective, and observe that users in different geo-social circles have various correlation strength.

- We suggest various correlation measures to capture the geo-social correlations of a user's check-in behavior on the cold-start problem, and determine the most effective correlation measures for each geo-social circle.

- We propose a geo-social correlation model (gSCorr) to solve the cold-start location recommendation problem by considering four types of geo-social circles with corresponding correlation strength.

The remainder of this paper is organized as follows. We first introduce the concept of geo-social correlations of check-in behavior on LBSNs in Section 2, present the proposed model for geo-social correlations in Section 3, discuss the experimental design and results on the real-world dataset in Section 4, followed by related work in Section 5, and provide some conclusions with future work in Section 6.

\section{Geo-Social Correlations on LBSNs}

When we observe a check-in from a user, there are two scenarios: checking in at a previous visited location, or a new location that the user has never checked 
in before. In this paper, we define the former one as "existing check-in(s)", and the latter one as "cold-start' check-in(s)", with respect to a user's checkin history. In 15, the authors explored the social-historical ties of check-in behavior on LBSNs, and found that both ties have effects on explaining a user's check-in behavior. To investigate the social correlations on a user's check-in behavior, we need a controlled social environment that excludes the effects of users' historical ties. However, to distinguish whether a user's existing check-in is correlated to his historical ties or social ties is actually a big challenge [15, while on the other hand, when a user performs a cold-start check-in, the effect of this behavior is more likely from his social ties than his historical ties, which indicates the chance to study the correlation between such check-ins and his social networks, while in turn also provides a feasible perspective of solving the traditional cold-start location recommendation problem. Therefore, we focus on investigating the social correlations with a user's cold-start check-ins by eliminating the historical tie effect to the largest extent.

Figure 2(a) shows the percentage of cold-start check-ins over the total number of observed check-ins in a period of a half year (January 1, 2011 to June 30, 2011) with 11,326 users and 1,171,521 check-ins on Foursquare (more details about this dataset in Section 4.1). The $\mathrm{x}$-axis represents the number of observed check-ins in a chronological order, and the $\mathrm{y}$-axis represents the percentage of cold-start check-ins. There are around $50 \%$ cold-start checkins within $2 \times 10^{5}$ observed check-ins, and around $35 \%$ "cold-start" check-ins among $1.2 \times 10^{6}$ observed check-ins, indicating that a user would like to go to a new location when he does not have much check-in history at early time; and then, as time goes by, the user would gradually shift his check-ins from new locations to existing locations. Furthermore, the high cold-start check-in ratio suggests cold-start check-ins take a big proportion of a user's check-in behavior. With half-year check-in history, a user would still have approximated one third probability to perform a cold-start check-in. Therefore, capturing a user's cold-start check-in location is necessary for designing improving location recommendation services.

Social scientists found that geographical distance plays an important role in social connections [24,18,6]. Previous work on LBSNs studied the spatial property of social networks, and reported that the probability of having a social connection between two individuals is a function of their distance [30. Therefore, to study the social correlation of a user's cold-start check-in behavior, we divide the social correlations into four sub-correlations, namely geo-social correlations, corresponding to four social circles with respect to the factors of social friendship and geographical distance. The confusing matrix of the four social circles is listed in Table 1 where $F$ indicates observed social friendship, $\bar{F}$ indicates non-friendship, $D$ indicates long geographical distance, and $\bar{D}$ indicates short geographical distance.

- $S_{F \bar{D}}$ : user's social circle consisting of his friends who live close;

- $S_{F D}$ : user's social circle consisting of his friends who live distant;

- $S_{\bar{F} \bar{D}}$ : user's social circle consisting of non-friend users who live close; and 
Table 1 Geo-Social Correlations

\begin{tabular}{l|c|c|}
\multicolumn{2}{c}{$\mathrm{F}$} & \multicolumn{1}{c}{$\bar{F}$} \\
\cline { 2 - 3 } $\mathrm{D}$ & $S_{F \bar{D}}:$ Local Friends & $S_{\bar{F} \bar{D}}:$ Local Non-friends \\
\cline { 2 - 3 } $\mathrm{D}$ & $S_{F D}:$ Distant Friends & $S_{\bar{F} D}:$ Distant Non-friends \\
\cline { 2 - 3 } & &
\end{tabular}

- $S_{\bar{F} D}$ : user's social circle consisting of non-friend users who live distant.

We define the four social circles as "geo-social circles". In [9], it is reported that the relative influence of a friend who lives $1,000 \mathrm{~km}$ away is 10 times greater than the influence of a friend who lives $40 \mathrm{~km}$ away on a user making check-ins. Therefore in this paper, we consider a pair of users within the same state/province as living close with short geographical distance, and a pair of users in different states/provinces as living distant with long geographical distance.

Figure 11illustrates a user's cold-start check-in behavior in different social correlation aspects. User $u$ goes to the airport at $t_{1}$, and then the restaurant at $t_{2}$ followed by the hospital at $t_{3}$. When $u$ performs a cold-start check-in at $t_{4}$, i.e., the check-in location does not belong to $\left\{l_{1}, l_{2}, l_{3}\right\}$, then it may be correlated to those users that are from $u$ 's different geo-social circles $S_{F \bar{D}}$, $S_{F D}, S_{\bar{F} \bar{D}}$ and $S_{\bar{F} D}$.

The investigation of geo-social correlations between a user's cold-start check-in behavior and the four geo-social circles, i.e., $S_{F \bar{D}}, S_{F D}, S_{\bar{F} \bar{D}}$ and $S_{\bar{F} D}$, enables us to study a user's check-in behavior in four aspects. The geosocial circle $S_{F \bar{D}}$ captures a user's local social correlations, sometimes also including local influence, such as going out with friends, or following friends' recommendations. The geo-social circle $S_{F D}$ captures a user's distant social correlations, such as visiting friends in another state. The third geo-social circle, $S_{\bar{F} \bar{D}}$, indicates that a user goes to a place where his local non-friends usually go to, usually referred to as "confounding" effect [10]. The last geosocial circle, i.e., $S_{\bar{F} D}$, suggests that a user would randomly visit some new locations due to an unknown effect regardless of what his friends or local users do. This could be, for example, visiting famous points of interest. Note that there could be some cold-start check-ins that cannot be correlated to any of the four geo-social circles. In our foursquare data, we found that such kind of cold-start check-ins only correspond to a small proportion (to discuss later in Table 3), therefore we consider it as an unknown effect and combine it to $S_{\bar{F} D}$ as well.

\section{Modeling Geo-Social Correlations}

\subsection{Problem Formulation}

To model the geo-social correlations of a user's cold-start check-in behavior, we consider the probability of a user $u$ checking-in at a new location $l$ at time $t$ as $P_{u}^{t}(l)$. With the four geo-social circles defined above, we further define 


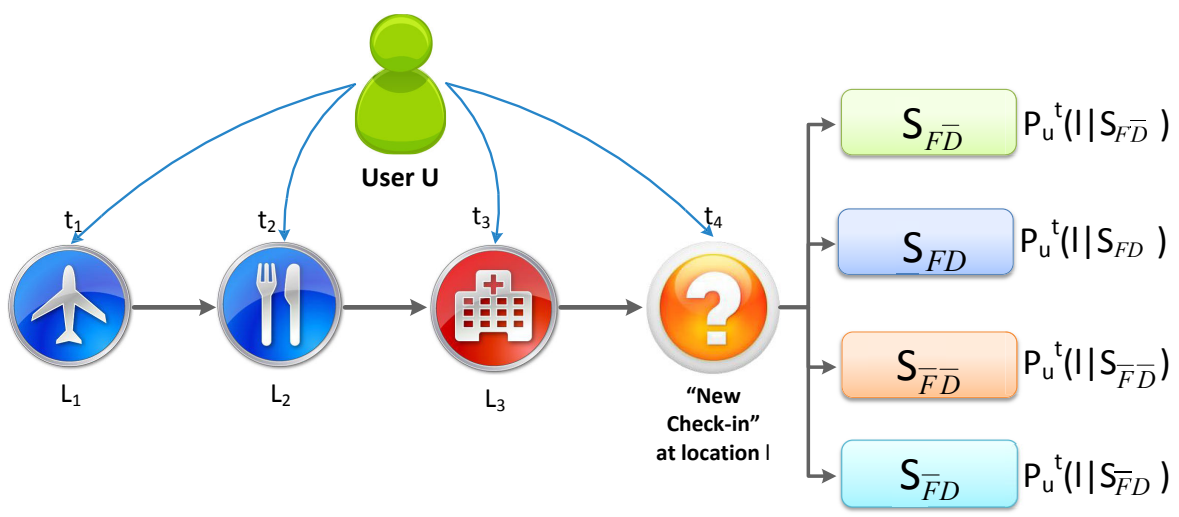

Fig. 1 The Geo-Social Correlations of cold-start Check-in Behavior

this probability as a combination of the four geo-social correlations,

$$
\begin{aligned}
& P_{u}^{t}(l)=\Phi_{1} P_{u}^{t}\left(l \mid S_{\bar{F} \bar{D}}\right)+\Phi_{2} P_{u}^{t}\left(l \mid S_{F \bar{D}}\right) \\
& +\Phi_{3} P_{u}^{t}\left(l \mid S_{F D}\right)+\Phi_{4} P_{u}^{t}\left(l \mid S_{\bar{F} D}\right) .
\end{aligned}
$$

where $\Phi_{1}, \Phi_{2}$ and $\Phi_{3}$ and $\Phi_{4}$ are correlation strength of different geo-social correlations, $P_{u}^{t}\left(l \mid S_{x}\right)$ indicates the geo-social correlation probability, which is the probability of user $u$ checking-in at location $l$ that is correlated to $u$ 's geo-social circle $S_{x}$. For example, $P_{u}^{t}\left(l \mid S_{F D}\right)$ indicates the probability of user $u$ checking-in at $l$ that is correlated to $u$ 's distant friends. In the following sections, we will further discuss how to model the geo-social correlation strength and correlation probabilities.

\subsection{Modeling Geo-Social Correlation Strength}

To explicitly model the correlation strength $\Phi_{1}, \Phi_{2}, \Phi_{3}$ and $\Phi_{4}$, we investigate the intrinsic patterns of correlations between a user's check-ins and his geosocial circles. We plot the percentage of cold-start check-ins that can be found from the different geo-social circles versus the total number of observed coldstart check-ins in Figure 2(b), with the same data set used in Figure 2(a). The $\mathrm{x}$-axis represents the number of observed cold-start check-ins in a chronological order, and the y-axis represents the percentage of cold-start check-in locations that have been checked-in before by users from that specific geo-social circle. For example, the blue line represents the percentage of cold-start check-in locations that have been visited by the user's local non-friends before. The percentage of cold-start check-ins from $S_{\bar{F} D}$ is not presented, since it can be deduced from the other three. Note that the geo-social correlations of the four geo-social circles may overlap. For example, a user may visit a new location $l$ where both of his local friends and distant friends have visited before. 


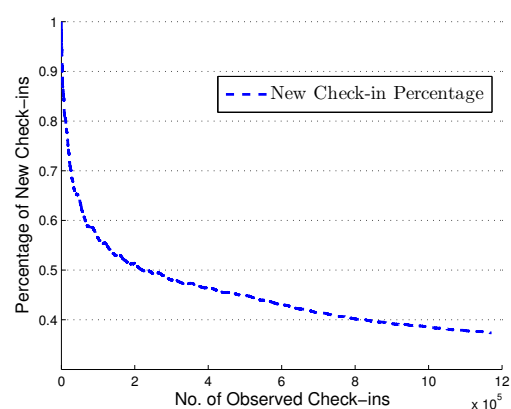

(a) The Ratio of cold-start Check-ins

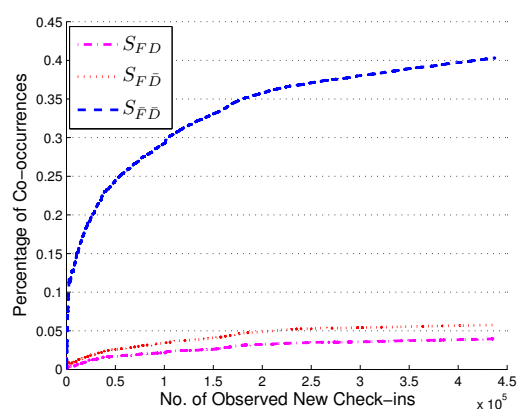

(b) Observed Social Correlations on coldstart Check-ins.

Fig. 2 The cold-start check-in rate and social correlation on Foursquare Data.

Eq. (1) indicates that with probability $\Phi_{1}$, the current cold-start check-in is correlated to $S_{\bar{F} \bar{D}}$. According to the observation in Figure 2(b), the correlation between cold-start check-ins and the geo-social circle $S_{\bar{F} \bar{D}}$ (blue line) increases with the increment of the number of observed cold-start check-ins. It keeps increasing rapidly early on, and then gradually becomes stable. The reason for this trend may come from two parts: (1) user $u$ would like to go to new locations when he does not have many historical check-ins, therefore in the early time, a lot of cold-start check-ins correlated to $S_{\bar{F} \bar{D}}$ are observed; and (2) as time goes by, the number of check-ins from $u$ 's geo-social circle is also increasing, which provides opportunities of co-occurrent check-ins between $u$ and his geo-social circle, hence the social correlation keeps increasing. Therefore, we set $\Phi_{1}$ as an active function to control the social correlation strength from local nonfriend users, which considers a set of features capturing $u$ 's historical check-in behavior and his different geo-social circles.

$$
\Phi_{1}=f\left(\mathbf{w}^{T} \mathbf{f}_{u}^{t}+b\right), 0 \leq \Phi_{1} \leq 1
$$

where $\mathbf{f}_{u}^{t}$ is a check-in feature vector of a single user $u$ at time $t, \mathbf{w}$ is a vector of the weights of $\mathbf{f}_{u}^{t}$, and b controls the bias. In this work, we define a user's check-in and social features $\mathbf{f}_{u}^{t}$ in Table2, Note that $\mathbf{f}_{u}^{t}$ is time sensitive, where all the features in $\mathbf{f}_{u}^{t}$ are computed at time t, and $S_{F \bar{D}}, S_{F D}$, and $S_{\bar{F} \bar{D}}$ are related to user $u$ 's geo-social circles.

$f(\bullet)$ is a real-valued and differentiable function that guarantees the range of $\Phi_{1}$ limited in $[0,1]$. In this case, a sigmoid function is often used [2], which can approximately capture the observations about $S_{\bar{F} \bar{D}}$ in Figure 2(b).

$$
f\left(\mathbf{w}^{T} \mathbf{f}_{u}^{t}+b\right)=\frac{1}{1+e^{-\left(\mathbf{w}^{T} \mathbf{f}_{u}^{t}+b\right)}}
$$


Table 2 check-in and social features

\begin{tabular}{|c|c|}
\hline Features & Description \\
\hline$N^{c}$ & Number of check-ins in $u$ 's history \\
\hline$N^{n c}$ & Number of cold-start check-ins in $u$ 's history \\
\hline \hline$N_{F \bar{D}}$ & Number of friends in $S_{F \bar{D}}$ \\
\hline$N_{F \bar{D}}^{c}$ & Number of check-ins from $S_{F \bar{D}}$ \\
\hline$N_{F \bar{D}}^{u c}$ & Number of unique check-ins from $S_{F \bar{D}}$ \\
\hline$N_{F \bar{D}}^{v c}$ & Number of visited check-ins from $S_{F \bar{D}}$ \\
\hline$N_{F \bar{D}}^{u v c}$ & Number of visited unique check-ins from $S_{F \bar{D}}$ \\
\hline \hline$N_{F D}$ & Number of friends in $S_{F D}$ \\
\hline$N_{F D}^{c}$ & Number of unique check-ins from $S_{F D}$ \\
\hline$N_{F D}^{u c}$ & Number of visited check-ins from $S_{F D}$ \\
\hline$N_{F D}^{v c}$ & Number of users in $S_{\bar{F} \bar{D}}$ \\
\hline$N_{F \bar{D}}^{u v c}$ & Number of visited unique check-ins from $S_{F D}$ \\
\hline \hline$N_{\bar{F} \bar{D}}$ & Number of check-ins from $S_{\bar{F} \bar{D}}$ \\
\hline$N_{\bar{F} \bar{D}}^{c}$ & Number of unique check-ins from $S_{\bar{F} \bar{D}}$ \\
\hline$N_{\bar{F} \bar{D}}^{u c}$ & Number of visited check-ins from $S_{\bar{F} \bar{D}}$ \\
\hline$N_{\bar{F} \bar{D}}^{v c}$ & Number of visited unique check-ins from $S_{\bar{F} \bar{D}}$ \\
\hline$N_{\bar{F} \bar{D}}^{u v c}$ &
\end{tabular}

Similarly, we observe that the social correlations of $S_{F D}$ and $S_{F \bar{D}}$ are fairly constant in Figure 2(b), therefore we define,

$$
\begin{aligned}
& \Phi_{2}=\left(1-\Phi_{1}\right) \phi_{1} \\
& \Phi_{3}=\left(1-\Phi_{1}\right)\left(1-\phi_{1}\right) \phi_{2} \\
& \Phi_{4}=\left(1-\Phi_{1}\right)\left(1-\phi_{1}\right)\left(1-\phi_{2}\right),
\end{aligned}
$$

where $\phi_{1} \in[0,1], \phi_{2} \in[0,1]$ are two constants to govern the social correlation strength of local friends and distant friends respectively.

Based on above definitions, we can rewrite the probability $P_{u}^{t}(l)$ in Eq. (1) as below,

$$
\begin{aligned}
& P_{u}^{t}(l)=f\left(\mathbf{w}^{T} \mathbf{f}_{u}^{t}+b\right) P_{u}^{t}\left(l \mid S_{\bar{F} \bar{D}}\right) \\
& +\left(1-f\left(\mathbf{w}^{T} \mathbf{f}_{u}^{t}+b\right)\right) \phi_{1} P_{u}^{t}\left(l \mid S_{F \bar{D}}\right) \\
& +\left(1-f\left(\mathbf{w}^{T} \mathbf{f}_{u}^{t}+b\right)\right)\left(1-\phi_{1}\right) \phi_{2} P_{u}^{t}\left(l \mid S_{F D}\right) \\
& +\left(1-f\left(\mathbf{w}^{T} \mathbf{f}_{u}^{t}+b\right)\right)\left(1-\phi_{1}\right)\left(1-\phi_{2}\right) P_{u}^{t}\left(l \mid S_{\bar{F} D}\right) .
\end{aligned}
$$

\subsection{Modeling Geo-Social Correlation Probabilities}

In this section, we discuss the modeling of geo-social correlation probabilities, i.e., $P_{u}^{t}\left(l \mid S_{x}\right)$, representing the probability of user $u$ checking in at location $l$ at time $t$ that is correlated to $u$ 's social circle $S_{x}, S_{x}=\left\{S_{F D}, S_{F \bar{D}}, S_{\bar{F} D}, S_{\bar{F} \bar{D}}\right\}$.

Gao et al. 15] reported that check-in sequence and text sentence share a large number of common properties, where a check-in location can be analog to a word. Thus, inspired by the "TF-IDF" strategy which is commonly used in text mining and information retrieval to determine the importance of a 
word, we propose location frequency (LF), user frequency (UF), and LF.UF correspondingly. The underlying assumption of "LF" is that a user tends to go to a place where his friend usually goes to. Previous work has also reported such property that the number of check-ins previously made by friends of a user is a good predictor for the user's next check-in location [7. Furthermore, to consider the uncertainty of normalization, we also propose a normalized version of "LF", i.e., "NLF". On the other hand, the underlying assumption of "UF" is that a user tends to go to a place where many of his friends have been to, which can be considered as another way to determine the importance of check-in location.

In [9], user similarity is also considered as important to explain the user's check-in behavior as different friendship may present different behavior similarity. In this work, to examine the probability $P_{u}^{t}\left(l \mid S_{x}\right)$, we first propose 5 geo-social correlation measures according to LF and UF without considering user similarity, and then propose another 5 geo-social correlations measures with user similarity accordingly, as described below,

- Location Frequency (LF)

A user may go to a new location that has been frequently visited by his geosocial circle before, therefore we define the probability of a user $u$ checking at location $l$ at time $t$ that is correlated with his geo-social circle $\mathcal{S}_{x}$ as:

$$
P_{u}^{t}\left(l \mid \mathcal{S}_{x}\right)=\frac{\sum_{v \in \mathcal{S}_{x}} N_{v}^{t}(l)}{\sum_{v \in \mathcal{S}_{x}} N_{v}^{t}}
$$

where $N_{v}^{t}(l)$ represents the number of check-ins at location $l$ by user $v$ before time $t$, and $N_{v}^{t}$ the total number of locations visited by user $v$ that user $u$ has not visited before time $t$.

\section{- Normalized Location Frequency (NLF)}

Normalized Location Frequency (NLF) calculates the ratio of check-ins at location $l$ for each user in $S_{x}$, and then normalized by the total number of users. The purpose of introducing this normalized measure of $\mathbf{L F}$ is due to the uncertainty of normalization in improving performance of user behavior modeling 35 .

$$
P_{u}^{t}\left(l \mid \mathcal{S}_{x}\right)=\frac{\sum_{v \in \mathcal{S}_{x}} \frac{N_{v}^{t}(l)}{N_{v}^{t}}}{N_{S_{x}}},
$$

where $N_{S_{x}}$ represents the number of users in $S_{x}$.

\section{- User Frequency (UF)}

User Frequency (UF) computes the probability $P_{u}^{t}\left(l \mid \mathcal{S}_{x}\right)$ as the ratio of users in $\mathcal{S}_{x}$ who have checked-in at $l$,

$$
P_{u}^{t}\left(l \mid \mathcal{S}_{x}\right)=\frac{\sum_{v \in \mathcal{S}_{x}} \delta_{v}^{t}(l)}{N_{\mathcal{S}_{x}}},
$$

where $\delta_{v}^{t}(l)$ equals to 1 if user $v$ has checked-in at $l$ before $t$, and 0 otherwise. 


\section{- Location Frequency \& User Frequency (LF.UF)}

As reported in [15], location sequences and document segments share a lot of common features. Traditional language model on language processing also achieves good performance when applied to the location prediction task. Therefore, inspired by the Tf-idf (a state-of-the-art weighting strategy widely used in language processing and information retrieval), we propose a LF.UF strategy to explore the geo-social correlations on LBSNs.

$$
P_{u}^{t}\left(l \mid \mathcal{S}_{x}\right)=\frac{\sum_{v \in \mathcal{S}_{x}} N_{v}^{t}(l)}{\sum_{v \in \mathcal{S}_{x}} N_{v}^{t}} \cdot \frac{\sum_{v \in \mathcal{S}_{x}} \delta_{v}(l)}{N_{\mathcal{S}_{x}}},
$$

- Normalized Location Frequency \& User Frequency (NLF.UF) Similar to the LF.UF measure, NLF.UF is defined as,

$$
P_{u}^{t}\left(l \mid \mathcal{S}_{x}\right)=\frac{\sum_{v \in \mathcal{S}_{x}} \frac{N_{v}^{t}(l)}{N_{v}^{t}}}{N_{S_{x}}} \cdot \frac{\sum_{v \in \mathcal{S}_{x}} \delta_{v}(l)}{N_{\mathcal{S}_{x}}},
$$

To integrate the effect of user similarities, we further propose another five measures that consider user similarities, corresponding to the five measures above.

- Sim-Location Frequency (S.LF)

$$
P_{u}^{t}\left(l \mid \mathcal{S}_{x}\right)=\frac{\sum_{v \in \mathcal{S}_{x}} s(u, v) N_{v}^{t}(l)}{\sum_{v \in \mathcal{S}_{x}} s(u, v) N_{v}^{t}},
$$

where $s(u, v)$ represents the user similarity between user $u$ and user $v$.

- Sim-Normalized Location Frequency (S.NLF)

$$
P_{u}^{t}\left(l \mid \mathcal{S}_{x}\right)=\frac{\sum_{v \in \mathcal{S}_{x}} s(u, v) \frac{N_{v}^{t}(l)}{N_{v}^{t}}}{\sum_{v \in \mathcal{S}_{x}} s(u, v)},
$$

- Sim-User Frequency (S.UF)

$$
P_{u}^{t}\left(l \mid \mathcal{S}_{x}\right)=\frac{\sum_{v \in \mathcal{S}_{x}} \delta_{v}(l) s(u, v)}{\sum_{v \in \mathcal{S}_{x}} s(u, v)},
$$

- Sim-Location Frequency \& User Frequency (S.LF.UF)

$$
P_{u}^{t}\left(l \mid \mathcal{S}_{x}\right)=\frac{\sum_{v \in \mathcal{S}_{x}} s(u, v) N_{v}^{t}(l)}{\sum_{v \in \mathcal{S}_{x}} s(u, v) N_{v}^{t}} \frac{\sum_{v \in \mathcal{S}_{x}} \delta_{v}(l)}{N_{\mathcal{S}_{x}}},
$$

- Sim-Normalized Location Frequency \& User Frequency (S.NLF.UF)

$$
P_{u}^{t}\left(l \mid \mathcal{S}_{x}\right)=\frac{\sum_{v \in \mathcal{S}_{x}} s(u, v) \frac{N_{v}^{t}(l)}{N_{v}^{t}}}{\sum_{v \in \mathcal{S}_{x}} s(u, v)} \frac{\sum_{v \in \mathcal{S}_{x}} \delta_{v}(l)}{N_{\mathcal{S}_{x}}} .
$$


In our model, the correlation probability from each circle, i.e., $P_{u}^{t}\left(l \mid \mathcal{S}_{\bar{F} \bar{D}}\right)$, $P_{u}^{t}\left(l \mid \mathcal{S}_{F \bar{D}}\right), P_{u}^{t}\left(l \mid \mathcal{S}_{F D}\right)$, and $P_{u}^{t}\left(l \mid \mathcal{S}_{\bar{F} D}\right)$, can be calculated with various measures. Due to the different user and check-in distributions of each geo-social circle, the measures may perform variously. Therefore measure selection is necessary for each sub-correlation to achieve a better model performance. We'll discuss the measure performance and selection in the experiment section.

\subsection{Parameter Inference}

With the definitions described in the last section, we discuss the process of inferring the parameters defined in Eq. (5). We define $(u, l, t)$ as a checkin action at location $l$ performed by user $u$ at time $t$, the likelihood of the observation over the whole data set is the product of the probability of each $(u, l, t)$ action, defined as:

$$
P(\mathcal{C} \mid \Theta)=\prod_{(u, l, t) \in \mathcal{C}} P_{u}^{t}(l),
$$

where $\mathcal{C}$ is the set of all the observed $(u, l, t)$ actions, and $\Theta$ is the parameter set consisting of $\mathbf{w}, b, \phi_{1}, \phi_{2}$. We learn these parameters through maximum likelihood, which is equivalent to the following minimization problem:

$$
\begin{aligned}
& \min \sum_{(u, l, t) \in \mathcal{C}}-\ln P(\mathcal{C} \mid \Theta) \\
& +\lambda\left(\|\mathbf{w}\|_{2}^{2}+\|b\|_{2}^{2}+\left\|\phi_{1}\right\|_{2}^{2}+\left\|\phi_{2}\right\|_{2}^{2}\right)
\end{aligned}
$$

where parameter $\lambda$ controls the quadratic regularized term to avoid overfitting. In this paper, we set the value of $\lambda$ as 0.05 , and get the objective function below,

$$
\begin{aligned}
& \min \sum_{(u, l, t) \in \mathcal{C}}-\ln \left(f\left(\mathbf{w}^{T} \mathbf{f}_{u}^{t}+b\right) P_{u}^{t}\left(l \mid S_{\bar{F} \bar{D}}\right)\right. \\
& +\left(1-f\left(\mathbf{w}^{T} \mathbf{f}_{u}^{t}+b\right)\right) \phi_{1} P_{u}^{t}\left(l \mid S_{F \bar{D}}\right) \\
& +\left(1-f\left(\mathbf{w}^{T} \mathbf{f}_{u}^{t}+b\right)\right)\left(1-\phi_{1}\right) \phi_{2} P_{u}^{t}\left(l \mid S_{F D}\right) \\
& \left.+\left(1-f\left(\mathbf{w}^{T} \mathbf{f}_{u}^{t}+b\right)\right)\left(1-\phi_{1}\right)\left(1-\phi_{2}\right) P_{u}^{t}\left(l \mid S_{\bar{F} D}\right)\right) \\
& +\lambda\left(\|\mathbf{w}\|_{2}^{2}+\|b\|_{2}^{2}+\left\|\phi_{1}\right\|_{2}^{2}+\left\|\phi_{2}\right\|_{2}^{2}\right) \\
& \text { s.t. } \quad 0 \leq \phi_{1} \leq 1, \quad 0 \leq \phi_{2} \leq 1
\end{aligned}
$$

We take the projected gradient method [5] to solve Eq. (18). The basic idea is to update each current parameter towards an optimal direction (determined by the first derivative of the objective function) with an appropriate step size in each learning step. In each step, if the parameter value runs out of the constraints (e.g., $0 \leq \phi_{1} \leq 1,0 \leq \phi_{2} \leq 1$ ), we project it back to the 
corresponding range. The process will go iteratively to update the parameters until convergence. As shown below, the parameters are updated as,

$$
\begin{aligned}
\mathbf{w} & \leftarrow \mathbf{w}-\gamma_{\mathbf{w}} \nabla_{\mathbf{w}} \\
b & \leftarrow b-\gamma_{b} \nabla_{b} \\
\phi_{1} & \leftarrow \begin{cases}0 & \phi_{1}-\gamma_{\phi_{1}} \nabla_{\phi_{1}}<0 \\
1 & \phi_{1}-\gamma_{\phi_{1}} \nabla_{\phi_{1}}>1 \\
\phi_{1}-\gamma_{\phi_{1}} \nabla_{\phi_{1}} & \text { else }\end{cases} \\
\phi_{2} & \leftarrow \begin{cases}0 & \phi_{2}-\gamma_{\phi_{2}} \nabla_{\phi_{2}}<0 \\
1 & \phi_{2}-\gamma_{\phi_{2}} \nabla_{\phi_{2}}>1 \\
\phi_{2}-\gamma_{\phi_{2}} \nabla_{\phi_{2}} & \text { else }\end{cases}
\end{aligned}
$$

where $\gamma_{\mathbf{w}}, \gamma_{b}, \gamma_{\phi_{1}}$ and $\gamma_{\phi_{2}}$ are learning step sizes, which are chosen to satisfy Goldstein Conditions [26]. $\nabla_{\mathbf{w}}, \nabla_{b}, \nabla_{\phi_{1}}$ and $\nabla_{\phi_{2}}$ are the partial derivatives of the objective function in Eq. (18) with respect to $\mathbf{w}, b, \phi_{1}$ and $\phi_{2}$ respectively,

$$
\begin{aligned}
& \nabla \mathbf{w}=2 \lambda \mathbf{w}-\sum_{(u, l, t) \in \mathcal{C}} \frac{B}{A} \frac{e_{1}}{\left(1+e_{1}\right)^{2}} \mathbf{f}_{u}^{t} \\
& \nabla b=2 \lambda b-\sum_{(u, l, t) \in \mathcal{C}} \frac{B}{A} \frac{e_{1}}{\left(1+e_{1}\right)^{2}} \\
& \nabla \phi_{1}=2 \lambda \phi_{1}-\sum_{(u, l, t) \in \mathcal{C}} \frac{\left(1-\Phi_{1}\right)}{A} C \\
& \nabla \phi_{2}=2 \lambda \phi_{2}-\sum_{(u, l, t) \in \mathcal{C}} \frac{\left(1-\Phi_{1}\right)\left(1-\phi_{1}\right)}{A} D
\end{aligned}
$$

where

$$
\begin{aligned}
& e_{1}=e^{-\left(\mathbf{w}^{T} \mathbf{f}_{u}^{t}+b\right)} \\
& A=\Phi_{1} P_{u}^{t}\left(l \mid S_{\bar{F} \bar{D}}\right)+\left(1-\Phi_{1}\right) \phi_{1} P_{u}^{t}\left(l \mid S_{F \bar{D}}\right) \\
& +\left(1-\Phi_{1}\right)\left(1-\phi_{1}\right) \phi_{2} P_{u}^{t}\left(l \mid S_{F D}\right) \\
& +\left(1-\Phi_{1}\right)\left(1-\phi_{1}\right)\left(1-\phi_{2}\right) P_{u}^{t}\left(l \mid S_{\bar{F} D}\right), \\
& B=P_{u}^{t}\left(l \mid S_{\bar{F} \bar{D}}\right)-\phi_{1} P_{u}^{t}\left(l \mid S_{F \bar{D}}\right)-\left(1-\phi_{1}\right) \phi_{2} P_{u}^{t}\left(l \mid S_{F D}\right) \\
& -\left(1-\phi_{1}\right)\left(1-\phi_{2}\right) P_{u}^{t}\left(l \mid S_{\bar{F} D}\right) \\
& C=P_{u}^{t}\left(l \mid S_{F \bar{D}}\right)-\phi_{2} P_{u}^{t}\left(l \mid S_{F D}\right)-\left(1-\phi_{2}\right) P_{u}^{t}\left(l \mid S_{\bar{F} D}\right) \\
& D=P_{u}^{t}\left(l \mid S_{F D}\right)-P_{u}^{t}\left(l \mid S_{\bar{F} D}\right)
\end{aligned}
$$

\section{Experiments}

In this work, we use location prediction to evaluate our proposed geo-social correlation model (gSCorr) 3 . In particular, we evaluate the following: (1) how

3 The code can be downloaded at http://www.public.asu.edu/^hgao16/code/gSCorr.zip 
well the proposed geo-social correlation measures capture the geo-social correlation probabilities; (2) how the geo-social correlation strengths and measures affect the cold-start check-in behavior; and (3) whether social correlations help cold-start location recommendation. Before we delve into experiment details, we first discuss an LBSN dataset and experiment settings.

\subsection{Data Collection}

We use a Foursquare dataset 4 to study the geo-social correlations of checkin behavior on location-based social networks. Foursquare is one of the most popular online LBSNs. It has more than 20 million users and 2 billion checkins as of April, 20125. The web site itself does not provide a public API to access users' check-in data, however, it provides an alternative way for users to link their twitter accounts with Foursquare, and then pop out the check-in messages as tweets to Twitter. Previous work [30,15] uses this way to collect the data from Twitter for studying check-in behavior. Similarly, by getting access to the check-in tweets through the Twitter REST API, we collected public Foursquare check-in data from January 2011 to December 2011. We also collected the user friendships and hometown information through Foursquare. Note that the friendships on Foursquare are undirected. The statistics of the final dataset are shown in Table 3. The user distributions w.r.t. the world and the USA are given in Figure $3(\mathrm{a})$ and Figure $3(\mathrm{~b})$ respectively.

\subsection{Experiment Setup}

We test our proposed model gSCorr on the data of each month from July to December respectively, with the corresponding training data from the previous 6 months to learn our model parameters as in Eq. (19). For example, when testing gSCorr on September data, we use the data from March to August to train our model.

For each month from July to December, we construct its test set and ground truth based on the observation of their corresponding cold-start check-in distributions in four geo-social circles. Table 4 lists detailed statistical information of the observed cold-start check-in distribution in four geo-social circles on the check-in data in July. Due to the space limit, we do not present the statistical information from the other months since they all have the similar distributions. We define "Social Co-occurrence Check-ins" (SCCs) as the cold-start check-ins whose check-in locations can be found from the user's different social circles before its checking in time. The check-in data in July contains 213,702 check-ins, with 77,581 cold-start check-ins performed at the locations that have never been visited before (the July test data is a closed set in the sense that it does not consider the historical check-ins before July,

\footnotetext{
4 The dataset is publically available at http://www.public.asu.edu/ hgao16/dataset.html

5 https://foursquare.com/about/
} 


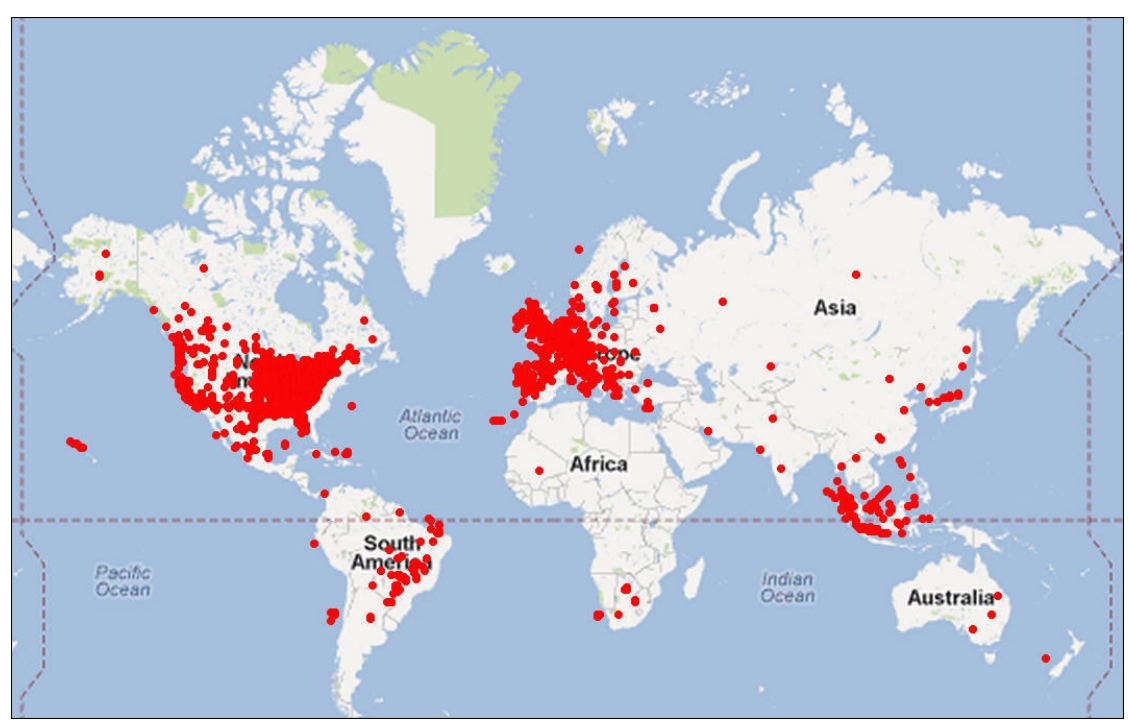

(a) The user distribution over the world.

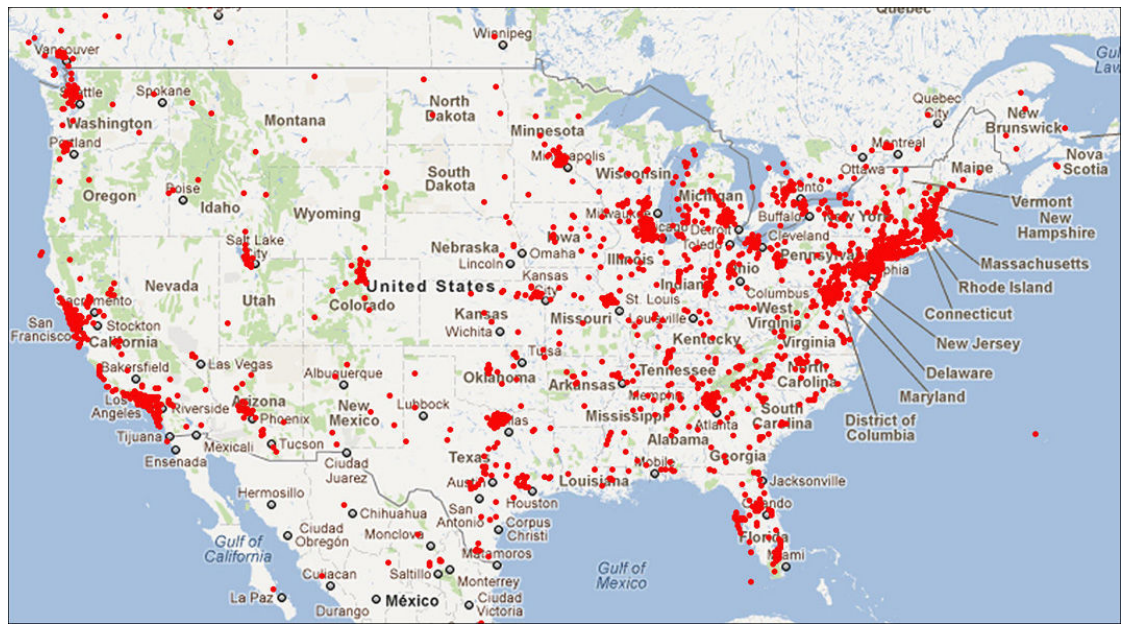

(b) The user distribution over the USA.

Fig. 3 The User Distribution on Foursquare.

as the same as the test data from other months). Among the 77,581 cold-start check-ins, around $44.5 \%$ SCCs can be found from the $S_{\bar{F} \bar{D}}, 7.26 \%$ from $S_{F \bar{D}}$, $4.62 \%$ from $S_{F D}$ and $50.82 \%$ from $S_{\bar{F} D}$. Only $10.61 \%$ SCCs are from a user's direct friendship circle. In other words, only 8,235 among 77,581 cold-start check-ins co-occurred with check-ins of the user's friendships. $S_{\bar{F} \bar{D}}$ has a large proportion of co-occurrences, indicating that user would like to go to a new location where his local non-friends in the state usually go. The number of SCCs 
Table 3 Statistical information of the dataset

\begin{tabular}{|c|c|}
\hline Duration & Jan 1, 2011-Dec 31, 2011 \\
\hline No. of users & 11,326 \\
\hline No. of check-ins & $2,290,997$ \\
\hline No. of unique locations & 187,218 \\
\hline No. of links & 47,164 \\
\hline Average check-ins per user & 202 \\
\hline Clustering coefficient & 0.1560 \\
\hline Average degree & 8.33 \\
\hline
\end{tabular}

Table 4 Statistical information of the July data

\begin{tabular}{|c|c|c|}
\hline Social Circle & No. of SCCs & Ratio \\
\hline$S_{\bar{F} \bar{D}}$ & 34,523 & $44.50 \%$ \\
\hline$S_{F \bar{D}}$ & 5,636 & $7.26 \%$ \\
\hline$S_{F D}$ & 3,588 & $4.62 \%$ \\
\hline$S_{\bar{F} D}$ & 39,423 & $50.82 \%$ \\
\hline Others & 1,672 & $2.2 \%$ \\
\hline$S_{\bar{F} \bar{D}} \cup S_{F \bar{D}}$ & 35,277 & $45.47 \%$ \\
\hline$S_{\bar{F} \bar{D}} \cup S_{F D}$ & 35,784 & $46.12 \%$ \\
\hline$S_{F \bar{D}} \cup S_{F D}$ & 8,235 & $10.61 \%$ \\
\hline$S_{\bar{F} \bar{D}} \cup S_{F \bar{D}} \cup S_{F D}$ & 36,486 & $47.03 \%$ \\
\hline
\end{tabular}

of $S_{\bar{F} \bar{D}} \cup S_{F \bar{D}} \cup S_{F D}$ doesn't increase much compared to $S_{\bar{F} \bar{D}}$, indicating that local non-friends have already covered most of the co-occurrences. Finally, we found that more than $50 \%$ of SCCs are correlated to $S_{\bar{F} D}$, which is difficult to capture for location prediction as the unknown effect. Note that there are $2.2 \%$ "Others", indicating that at the time of check-in, 1,672 cold-start checkins cannot be found from any of the four social circles. We consider this as an unknown effect and merge it into $S_{\bar{F} D}$.

We use location recommendation to evaluate our correlation measures and model performance. The user similarities are computed based on the checkin data in the first half year by cosine similarity, while each user is represented by a check-in vector, and the entry in the vector indicates the visiting frequency of the user at the location. For each test month, the test set is selected as the SCCs of $S_{\bar{F} \bar{D}} \cup S_{F \bar{D}} \cup S_{F D}$, and the ground truth is the corresponding check-in locations. We do not consider $S_{\bar{F} D}$ because from a user's perspective, friends and local non-friends are the ones that are reachable, while the distant non-friend users are too weak in relation for the user to correlate.

\subsection{Geo-Social Correlation Measure Selection}

Before we discuss the performance of our proposed model gSCorr, we first evaluate the 10 geo-social correlation measures described in Section 3.3. Each measure can be directly applied to the test set and generates a ranking list of location probabilities $P_{u}^{t}\left(l \mid S_{x}\right)$ with respect to the geo-social circles. We select the location with the highest $P_{u}^{t}\left(l \mid S_{x}\right)$ as recommended location for the coldstart check-in, and evaluate the performance with accuracy. The purpose of 
this comparison is to select the best correlation measure for each geo-social circle, and utilize the most suitable ones for $P_{u}^{t}\left(l \mid S_{x}\right)$ in Eq. (11). The results are shown in Table 5. Table 6. and Table 7 with the best performance of each month in bold. We summarize several observations below:

- The user similarity consistently improves the recommendation performance. Comparing with measures without considering user similarities, measures with user similarities on average have $5.36 \%$ relative improvement on $S_{F \bar{D}}$, $30.53 \%$ relative improvement on $S_{F D}$, and $15.89 \%$ relative improvement on $S_{\bar{F} \bar{D}}$, suggesting that user similarity is a significant factor to capture human mobile behavior.

- The comparison of $\mathbf{L F}$ and its normalized version NLF (including those measures containing $\mathbf{L F}$ and $\mathbf{N L F}$ ) indicates that normalization does not always improve the performance, which is consistent to the findings in 35 . Depending on which social circle we apply the measure to, normalization may result in various performances in capturing the geo-social correlations.

- S.Lf.Uf is the best measure for capturing the social correlations of local friends $S_{F \bar{D}}$. It also performs well on the other two geo-social circles especially on $S_{F \bar{D}}$. It considers the user frequency, location frequency and user similarities together, and obtains $1 \%$ relative improvement compared to the second best rated (S.LF), and $24.88 \%$ relative improvement compared to the worst rated (Uf).

- S.Lf shows good performance in capturing the social correlations of distant friends $S_{F D}$. It considers the location frequency and user similarity without the user frequency. One possible reason of this may be due to the smaller number of distant friends (2.68 per user on average) compared with the number of local friends (5.64 per user on average), which makes it a weak measure by counting the user frequency of distant friends.

- The performance on $S_{\bar{F} \bar{D}}$ indicates that its best correlation measure is S.Uf, suggesting that a user would like to go to a location that has been visited by a large proportion of local non-friend users, no matter how frequently the location is visited by each individual user. This is consistent to the confounding effect that people who live in similar environment tend to share similar behavior, which is exactly the geo-social circle $S_{\bar{F} \bar{D}}$ supposed to capture.

Due to the varied performances of each correlation measure on each geo-social circle, we conclude that measure selection is necessary for computing geosocial correlation probabilities. Hence, we apply S.Lf.Uf, S.Lf and S.Uf to compute $P_{u}^{t}\left(l \mid S_{F \bar{D}}\right), P_{u}^{t}\left(l \mid S_{F D}\right)$ and $P_{u}^{t}\left(l \mid S_{\bar{F} \bar{D}}\right)$ respectively in the following experiments, considering their good performance on the corresponding geosocial circles. We do not report the results on $S_{\bar{F} D}$, since for the unknown effect $P_{u}^{t}\left(l \mid S_{\bar{F} D}\right)$, all the measures applied to $S_{\bar{F} D}$ perform as a random guess in our experiment, one possible reason may be the large number of users and candidate locations within this geo-social circle. Therefore, to reduce the time complexity, we consider $P_{u}^{t}\left(l \mid S_{\bar{F} D}\right)$ as a probability of a random jump to a location in current location vocabulary that user $u$ has not checked-in before. 
Table 5 Location Recommendation for Measure Selection on $S_{F \bar{D}}$

\begin{tabular}{|c|c|c|c|c|c|c|}
\hline \multirow{2}{*}{ Ranking Strategy } & \multicolumn{5}{|c|}{$S_{F \bar{D}}$} \\
\cline { 2 - 7 } & Jul & Aug & Sep & Oct & Nov & Dec \\
\hline LF & $5.85 \%$ & $6.24 \%$ & $6.49 \%$ & $6.76 \%$ & $6.47 \%$ & $7.29 \%$ \\
\cline { 2 - 7 } NLF & $5.60 \%$ & $6.07 \%$ & $6.11 \%$ & $6.42 \%$ & $6.32 \%$ & $6.88 \%$ \\
\cline { 2 - 7 } UF & $5.18 \%$ & $5.54 \%$ & $5.68 \%$ & $5.70 \%$ & $5.69 \%$ & $6.33 \%$ \\
LF.UF & $6.16 \%$ & $6.50 \%$ & $6.72 \%$ & $6.99 \%$ & $6.70 \%$ & $7.33 \%$ \\
NLF.UF & $5.92 \%$ & $6.39 \%$ & $6.49 \%$ & $6.78 \%$ & $6.58 \%$ & $7.22 \%$ \\
\cline { 2 - 7 } S.LF & $6.30 \%$ & $6.73 \%$ & $6.99 \%$ & $7.32 \%$ & $7.04 \%$ & $\mathbf{7 . 9 0 \%}$ \\
S.NLF & $5.89 \%$ & $6.31 \%$ & $6.34 \%$ & $6.64 \%$ & $6.62 \%$ & $7.21 \%$ \\
S.UF & $5.38 \%$ & $5.83 \%$ & $5.77 \%$ & $5.97 \%$ & $5.96 \%$ & $6.58 \%$ \\
\cline { 2 - 7 } S.LF.UF & $\mathbf{6 . 5 1 \%}$ & $\mathbf{6 . 8 5 \%}$ & $\mathbf{7 . 0 2 \%}$ & $\mathbf{7 . 3 7 \%}$ & $\mathbf{7 . 1 1 \%}$ & $7.76 \%$ \\
\cline { 2 - 7 } S.NLF.UF & $6.23 \%$ & $6.68 \%$ & $6.75 \%$ & $7.07 \%$ & $6.92 \%$ & $7.55 \%$ \\
\hline
\end{tabular}

Table 6 Location Recommendation for Measure Selection on $S_{F D}$

\begin{tabular}{|c|c|c|c|c|c|c|}
\hline \multirow{2}{*}{ Ranking Strategy } & \multicolumn{7}{|c|}{$S_{F D}$} \\
\cline { 2 - 7 } & Jul & Aug & Sep & Oct & Nov & Dec \\
\hline LF & $2.39 \%$ & $2.39 \%$ & $2.91 \%$ & $3.35 \%$ & $3.26 \%$ & $3.65 \%$ \\
\cline { 2 - 7 } NLF & $2.65 \%$ & $2.50 \%$ & $3.07 \%$ & $3.39 \%$ & $3.33 \%$ & $3.38 \%$ \\
UF & $2.23 \%$ & $2.22 \%$ & $2.66 \%$ & $3.15 \%$ & $3.03 \%$ & $3.15 \%$ \\
LF.UF & $2.65 \%$ & $2.70 \%$ & $3.20 \%$ & $3.66 \%$ & $3.52 \%$ & $3.59 \%$ \\
NLF.UF & $2.79 \%$ & $2.70 \%$ & $3.26 \%$ & $3.64 \%$ & $3.52 \%$ & $3.56 \%$ \\
\cline { 2 - 7 } S.LF & $\mathbf{3 . 6 5 \%}$ & $3.52 \%$ & $4.15 \%$ & $\mathbf{4 . 6 3 \%}$ & $\mathbf{4 . 3 7 \%}$ & $\mathbf{4 . 9 1 \%}$ \\
S.NLF & $3.45 \%$ & $3.46 \%$ & $3.92 \%$ & $4.31 \%$ & $4.14 \%$ & $4.40 \%$ \\
S.UF & $3.14 \%$ & $3.00 \%$ & $3.43 \%$ & $3.86 \%$ & $3.76 \%$ & $4.01 \%$ \\
S.LF.UF & $3.64 \%$ & $\mathbf{3 . 5 7 \%}$ & $\mathbf{4 . 1 9 \%}$ & $4.56 \%$ & $4.31 \%$ & $4.64 \%$ \\
\cline { 2 - 7 } S.NLF.UF & $3.58 \%$ & $3.52 \%$ & $4.11 \%$ & $4.47 \%$ & $4.25 \%$ & $4.47 \%$ \\
\hline
\end{tabular}

Table 7 Location Recommendation for Measure Selection on $S_{\bar{F} \bar{D}}$

\begin{tabular}{|c|c|c|c|c|c|c|}
\hline \multirow{2}{*}{ Ranking Strategy } & \multicolumn{7}{|c|}{$S_{\bar{F} \bar{D}}$} \\
\cline { 2 - 7 } & Jul & Aug & Sep & Oct & Nov & Dec \\
\hline LF & $14.42 \%$ & $15.47 \%$ & $15.34 \%$ & $16.17 \%$ & $16.32 \%$ & $18.70 \%$ \\
\cline { 2 - 7 } NLF & $15.23 \%$ & $16.30 \%$ & $16.35 \%$ & $17.32 \%$ & $17.45 \%$ & $19.67 \%$ \\
UF & $15.35 \%$ & $16.54 \%$ & $16.50 \%$ & $17.64 \%$ & $17.77 \%$ & $19.81 \%$ \\
\cline { 2 - 7 } LF.UF & $15.22 \%$ & $16.44 \%$ & $16.40 \%$ & $17.41 \%$ & $17.53 \%$ & $19.87 \%$ \\
NLF.UF & $15.44 \%$ & $16.59 \%$ & $16.58 \%$ & $17.66 \%$ & $17.77 \%$ & $19.84 \%$ \\
S.LF & $17.84 \%$ & $18.75 \%$ & $18.60 \%$ & $19.56 \%$ & $19.72 \%$ & $22.38 \%$ \\
S.NLF & $18.00 \%$ & $19.01 \%$ & $19.04 \%$ & $19.81 \%$ & $19.84 \%$ & $22.39 \%$ \\
S.UF & $\mathbf{1 8 . 3 7 \%}$ & $\mathbf{1 9 . 4 0 \%}$ & $\mathbf{1 9 . 4 5 \%}$ & $\mathbf{2 0 . 2 1 \%}$ & $\mathbf{2 0 . 3 4 \%}$ & $\mathbf{2 2 . 8 2 \%}$ \\
\cline { 2 - 7 } S.LF.UF & $17.75 \%$ & $18.86 \%$ & $18.80 \%$ & $19.74 \%$ & $20.10 \%$ & $22.34 \%$ \\
S.NLF.UF & $17.68 \%$ & $18.66 \%$ & $18.66 \%$ & $19.69 \%$ & $19.80 \%$ & $22.36 \%$ \\
\hline
\end{tabular}

\subsection{Performance of gSCorr}

In this section, we discuss the performance of gSCorr on cold-start checkin location recommendation problem with the correlation measures selected in the above section. Note that gSCorr is different from traditional recommendation approaches. The traditional recommendation methods, such as collaborative filtering, usually perform recommendation based on a single user-location matrix generated from training data, which ignore the temporal information among user's check-ins. While in gSCorr, for each test case (a cold-start 
check-in location in test set), only check-ins before the check-in time of test case are used for prediction. Of course, methods based on collaborative filtering can still be applied with the consideration of temporal information, by generating a user-location matrix considering only the previous user check-ins for each test case in the test set. We will use this approach as one baseline to compare with gSCorr in our experiment, and show its relationship to our proposed correlation measures.

We compare gSCorr with four baselines, one is from the observation of the measure selection in Table 5, Table 6] and Table 7 , the other three are selected as the existing most popular location recommendation model on LBSNs.

- S.LF.UF: We select S.LF.UF to capture the geo-social correlations and predict cold-start check-ins. It performs well on all the geo-social circles, and achieve the best performance on $S_{F \bar{D}}$ and many times on $S_{F \bar{D}}$. We apply it to the whole test set to predict the cold-start check-ins.

- Periodic \& Social Mobility Model (PSMM): PSMM ranks the locations based on a user's periodic and social patterns 9 . Since the periodic patterns can only recommend existing locations, we adopt the social patterns to recommmend the cold-start check-ins.

- Social-Historical Model (SHM): SHM integrates a user's historical ties and social ties to recommend/predict the next check-in location [15]. Similar to PSMM, we leverage the social model which utilizes the social ties to recommend cold-start check-in locations.

- Collaborative Filtering (CF): $\mathbf{C F}$ is a state-of-the-art approach for recommender systems. It computes a user's interest in a location based on other users' interests in that location. Since it can recommend new locations to a user, we apply it to each test case of our test set and consider that a correct recommendation happens when the recommended location is the same as the ground truth of the test case. We choose user-based collaborative filtering for such recommendation [33] as shown below:

$$
P_{u}^{t}(l)=\frac{\sum_{v \in \mathcal{U}} s(u, v) r_{v, l}}{\sum_{v \in \mathcal{U}} s(u, v)} .
$$

where $\mathcal{U}$ is the set of users who have visited $l, r_{v, l}$ is the preference of user $v$ on location $l$, which in our experiment is chosen as proportional to number of $v$ 's check-ins on $l$ normalized by $v$ 's total number of check-ins, i.e., $\frac{N_{v}^{t}(l)}{N_{v}^{t}}$.

The results are shown in Table 8 . The best performance of each month among all the approaches is in bold. We summarize several interesting observations below:

- Both PSMM and SHM do not perform well in recommending the coldstart check-in locations. SHM performs better than PSMM, but still only achieve a low accuracy. They recommend a user's next location based on the observation of his friends' check-in history. The performance indicates that a user does not follow his friends' check-in sequence a lot on LBSNs, especially when performing a cold-start check-in. 
Table 8 Performance Comparison for Location Recommendation

\begin{tabular}{|c|c|c|c|c|c|c|}
\hline Dataset & Jul & Aug & Sep & Oct & Nov & Dec \\
\hline S.LF.UF & $18.31 \%$ & $19.58 \%$ & $19.71 \%$ & $20.79 \%$ & $21.10 \%$ & $23.53 \%$ \\
\hline PSMM & $1.04 \%$ & $1.19 \%$ & $1.24 \%$ & $1.22 \%$ & $1.26 \%$ & $1.23 \%$ \\
\hline SHM & $5.30 \%$ & $5.08 \%$ & $5.39 \%$ & $5.65 \%$ & $5.03 \%$ & $5.58 \%$ \\
\hline CF & $18.24 \%$ & $19.57 \%$ & $19.45 \%$ & $20.74 \%$ & $20.84 \%$ & $23.59 \%$ \\
\hline gSCorr & $\mathbf{1 9 . 2 1 \%}$ & $\mathbf{2 0 . 2 5 \%}$ & $\mathbf{2 0 . 3 6 \%}$ & $\mathbf{2 1 . 2 6 \%}$ & $\mathbf{2 1 . 4 2 \%}$ & $\mathbf{2 4 . 1 3 \%}$ \\
\hline
\end{tabular}

Table 9 Evaluation Measures

\begin{tabular}{|c|c|c|}
\hline & Single Measure & Various Measures \\
\hline Equal Strength & EsSm & EsVm \\
\hline Random Strength & RsSm & RsVm \\
\hline Various Strength & VsSm & gSCorr \\
\hline
\end{tabular}

- CF has comparable performance with S.LF.UF. Comparing to Eq.(12), applying S.NLF.UF to the whole test set is actually equivalent to userbased collaborative filtering, resulting in a close performance to S.LF.UF according to Table 5, Table [6, and Table 7 This also demonstrates the practicability of our proposed correlation measures.

- gSCorr performs the best among all the approaches. To demonstrate the significance of its improvement over other baseline methods, we launch a random guess approach to recommend the cold-start check-ins. The recommendation accuracy of the random guess is always below $0.005 \%$, indicating that gSCorr significantly improves the baseline methods.

\subsection{Effect of Geo-Social Correlation Strength and Measures}

To further investigate gSCorr, we consider the effect of both geo-social correlation strength and measures in capturing the user's cold-start check-in behavior. Therefore, we set up five alternative approaches with various correlation strength and measures, to compare the location recommendation performance with gSCorr, as shown in Table 9 with the details below:

- EsSm. We select the measure S.LF.UF, which works well in all the geosocial circles, to compute the geo-social correlation probabilities for each social circle. We set all the geo-social correlation strength equaling to 1 .

- EsVm. We select the same measures as in gSCorr, but set all the geosocial correlation strength as 1 .

- RsSm. We select the same measure as in EsSm, and randomly assign the geo-social correlation strength.

- RsVm. We select the same measures as in gSCorr, and randomly assign the geo-social correlation strength.

- VsSm. We select the same measure as in EsSm, and perform the same training procedure to obtain the geo-social correlation strength.

Note that gSCorr is a various strength and various measures approach. The results are shown in Table 10 with the best performance of each month in 
Table 10 Location Recommendation with Various Geo-Social Correlation Strength and Measures

\begin{tabular}{|c|c|c|c|c|c|c|}
\hline Dataset & Jul & Aug & Sep & Oct & Nov & Dec \\
\hline EsVm & $17.88 \%$ & $18.60 \%$ & $18.86 \%$ & $19.48 \%$ & $19.64 \%$ & $21.94 \%$ \\
\hline EsSm & $16.20 \%$ & $16.86 \%$ & $17.11 \%$ & $17.94 \%$ & $18.16 \%$ & $20.57 \%$ \\
\hline VsSm & $16.49 \%$ & $17.94 \%$ & $18.08 \%$ & $18.17 \%$ & $18.45 \%$ & $20.90 \%$ \\
\hline RsSm & $14.93 \%$ & $15.49 \%$ & $15.88 \%$ & $16.70 \%$ & $16.97 \%$ & $19.29 \%$ \\
\hline RsVm & $15.23 \%$ & $15.78 \%$ & $16.17 \%$ & $16.81 \%$ & $17.02 \%$ & $19.10 \%$ \\
\hline gSCorr $(V s V m)$ & $\mathbf{1 9 . 2 1 \%}$ & $\mathbf{2 0 . 2 5 \%}$ & $\mathbf{2 0 . 3 6 \%}$ & $\mathbf{2 1 . 2 6 \%}$ & $\mathbf{2 1 . 4 2 \%}$ & $\mathbf{2 4 . 1 3 \%}$ \\
\hline
\end{tabular}

bold; For each random strength approach (RsSm and $\mathrm{RsVm}$ ), we run 30 times and report the average accuracy. We summarize the essential observations below:

- The geo-social correlations from different geo-social circles contribute variously to a user's check-in behavior. Both $\boldsymbol{V} \boldsymbol{s} \boldsymbol{S}$ and $\boldsymbol{g S C o r r}$ perform better than their equal strength versions (i.e., $\boldsymbol{E} \boldsymbol{s} \boldsymbol{S} \boldsymbol{m}$ and $\boldsymbol{E} \boldsymbol{s} \boldsymbol{V} \boldsymbol{m}$ ), respectively, indicating that the geo-social correlations are not equally weighted.

- The randomly assigned strength approaches ( $R \boldsymbol{s} \boldsymbol{S m}$ and $\boldsymbol{R} \boldsymbol{s} \boldsymbol{V} \boldsymbol{m})$ perform the worst comparing to the other approaches, where the average performance of $\boldsymbol{V s} \boldsymbol{S m}$ has a $9.40 \%$ relative improvement over $\boldsymbol{R} \boldsymbol{s} \boldsymbol{S m}$, and $\boldsymbol{g S C o r r}$ has a $27.51 \%$ relative improvement over $\boldsymbol{R} \boldsymbol{s} \boldsymbol{m}$, indicating that social correlation strength do affect the check-in behavior.

- The single measure approaches $(\boldsymbol{E} \boldsymbol{s} \boldsymbol{S m}, \boldsymbol{R} \boldsymbol{s} \boldsymbol{S m}, \boldsymbol{V} \boldsymbol{S} \boldsymbol{S m})$ always perform worse than the various measures approaches ( $\boldsymbol{E} \boldsymbol{s} \boldsymbol{V} \boldsymbol{m}, \boldsymbol{R} \boldsymbol{s} \boldsymbol{V}, \boldsymbol{g S C o r r})$, which suggests that for different social circles, there are different suitable correlation measures.

- gSCorr performs the best on all the test data, suggesting the advantage of gSCorr as considering different geo-social correlation strength and measures for each geo-social circle, which results in a flexible model for capturing the geo-social correlations on a user's check-in behavior.

\subsection{Effect of Different Geo-Social Circles}

To further investigate the contribution of different geo-social circles, we compare the recommendation results by utilizing various combinations of geosocial circles, as shown in Table 11] The geo-social correlation measures are all selected as the best one for the corresponding social circles, and the geo-social correlation strength is learned in the previous section through gSCorr.

The results show that the social correlations of user's direct friendships $S_{F D}$ and $S_{F \bar{D}}$ are significantly lower than the local non-friend users $S_{\bar{F} \bar{D}}$. The latter contributes more than $95 \%$ of accurate recommendation, which indicates that there is a big overlap of check-in locations between local non-friend users and direct friends. On the other hand, the correlations of $S_{F D}$ and $S_{F \bar{D}}$ do not overlap much, where the combination of them has significant improvement over $S_{F D}$ and $S_{F \bar{D}}$ individually. This is due to the diversity of friend distribution 
Table 11 Location Recommendation with Various Social Circle Combinations

\begin{tabular}{|c|c|c|c|c|c|c|}
\hline Methods & Jul & Aug & Sep & Oct & Nov & Dec \\
\hline$S_{F \bar{D}}$ & $6.51 \%$ & $6.85 \%$ & $7.02 \%$ & $7.37 \%$ & $7.11 \%$ & $7.76 \%$ \\
\hline$S_{F D}$ & $3.65 \%$ & $3.52 \%$ & $4.15 \%$ & $4.63 \%$ & $4.37 \%$ & $4.91 \%$ \\
\hline$S_{\bar{F} \bar{D}}$ & $18.37 \%$ & $19.40 \%$ & $19.45 \%$ & $20.21 \%$ & $20.34 \%$ & $22.82 \%$ \\
\hline$S_{\bar{F} \bar{D}} \cup S_{F \bar{D}}$ & $18.62 \%$ & $19.60 \%$ & $19.62 \%$ & $20.49 \%$ & $20.73 \%$ & $23.22 \%$ \\
\hline$S_{\bar{F} \bar{D}} \cup S_{F D}$ & $19.01 \%$ & $20.09 \%$ & $20.23 \%$ & $21.08 \%$ & $21.17 \%$ & $23.97 \%$ \\
\hline$S_{F \bar{D}} \cup S_{F D}$ & $8.33 \%$ & $8.46 \%$ & $9.04 \%$ & $9.43 \%$ & $9.23 \%$ & $10.09 \%$ \\
\hline$S_{\bar{F} \bar{D}} \cup S_{F \bar{D}} \cup S_{F D}$ & $\mathbf{1 9 . 2 1 \%}$ & $\mathbf{2 0 . 2 5 \%}$ & $\mathbf{2 0 . 3 6 \%}$ & $\mathbf{2 1 . 2 6 \%}$ & $\mathbf{2 1 . 4 2 \%}$ & $\mathbf{2 4 . 1 3 \%}$ \\
\hline
\end{tabular}

since local friends and distant friends do not share much common geographical environment. Furthermore, the combination of $S_{\bar{F} D} \cup S_{F \bar{D}}$ performs much better than $S_{\bar{F} D} \cup S_{F D}$, indicating that local non-friend users share more common check-in locations with local friends than distant friends. Finally, gSCorr always performs the best among all the combinations of social circles (in bold font), demonstrating that by taking advantage of both social networks and geographical distance, our approach properly captures the user's coldstart check-in behavior on LBSNs, and could be utilized to benefit cold-start location recommendation.

\subsection{Discussion}

We summarize the experiment results in this section, and explain a set of observations of user check-in behavior on LBSNs as below:

- Social correlations do exist on LBSNs. The correlation is more relevant to a user's local non-friends than direct social friends, where the latter only contribute a small proportion in a user's check-in behavior. This in turn explains the previous findings $9,15,7,36,37$, that utilizing social friends' check-ins can only slightly improve the location prediction/recommendation on LBSNs.

- To capture the social correlations on LBSNs, a set of factors need to be considered, which consists of user similarity, location frequency and user frequency. Furthermore, the factors affect variously on user's different geosocial circles.

- Social correlations can be utilized to solve the cold-start problem to a certain extent. From the results in Table 10 and 11, gSCorr could accurately recommend $19.21 \%$ cold-start check-in locations from $S_{\bar{F} \bar{D}} \cup S_{F \bar{D}} \cup S_{F D}$. Considering the total number of cold-start check-ins $(77,581)$ in the data set, it is equivalent to around $10 \%$ accuracy among the whole dataset, while a random guess of a user's next location from our testing set is below $0.005 \%$, indicating that the improvement of $\mathbf{g S C o r r}$ in recommending the cold-start location is actually very significant. 
4.8 Limitations of gSCorr

gSCorr considers both geographical distance and social friendships, providing a better perspective to compute user similarity for recommendation purpose. According to the comparison between gSCorr and state-of-the-art baseline methods, the geographical property does present significant effect in improving the location recommendation. However, there are several limitations of gSCorr that could be considered for future improvements.

\section{- Discrete Geographical Distance}

The geographical separation of social relationships in this work is discrete due to the limitation of data availability. According to the observed user profiles, hometown information is usually provided in city or state level. Considering geographical distance in state level may loss valuable local information, while adopting city level distance measure may result in the incorrect use of state level information. One possible way is to consider all the check-ins of a user and take the average; however this strategy is highly affected by the check-in outliers, i.e., check-in locations far away from the hometown. Thus, under which granularity to compute the geographical distance is still an open question. We will continue to study this problem and investigate an appropriate social correlation function of continuous geographical distance.

\section{- Temporal Dynamics of Check-in Behavior}

As human movement is a stochastic process over the time, the corresponding geo-social correlations may also change over the time. Adopting geosocial correlation measures analog to "TF-IDF" is under the assumption of "bag of check-ins", where check-in locations are independent to each other. This may result in the temporal information loss as the older check-in could have a decreasing correlation to the current check-in. We have performed preliminary experiments to evaluate such effect. The experimental results show that geo-social correlations do decrease over the time. In our observations, we have found that using the recent $30 \%$ check-ins in half-year duration from a user is sufficient to compute the user similarity and perform recommendation. This observation could be potentially utilized to improve our algorithm efficiency significantly, as human check-in sequence is usually too long to be efficiently leveraged in similarity computation.

\section{Related Work}

Researchers have investigated the social network and check-in properties on location-based social networks [12. Noulas et al. 27] studied the spatio-temporal patterns of user activity on Foursquare, and found that the check-in activity varies within the course of a day and a week. Long et al. 22 investigated the local geographical topics of check-ins with LDA, and studied the spatial and temporal properties of discovered topics. Cheng et al. 8 reported that user's check-in behavior on LBSNs follows the "Lèvy Flight" mobility pattern, and is 
influenced by the social status, sentiment and geographic constraints. Gao et al. [13, 14 investigated the temporal properties of geographical check-ins, and leverage them for location prediction and location recommendation. In [28, 30, the authors studied the correlation between friends and their average distance on three LBSNs, and observed that the probability of having a social connection between two users is a function of their geographical distance. In [3], the authors found that the probability of friendship is roughly inversely proportional to distance, and this information has been further studied to predict a user's home address with Facebook data.

Efforts have also been made to utilize user's social network information on LBSNs for improving location based services. In [7, the authors investigated various features for location prediction on LBSNs, and reported that the number of check-ins made by friends is a significant predictor. In [23], the authors proposed a HITS-based POI recommendation algorithm to recommend POIs to LBSN users with the consideration of social relationships. In [36], the checkin information from nearby friends was utilized for location recommendation while other users were ignored. The results indicate that social network only brings minor improvement. Gao et al. [16] investigated geo-social correlations on LBSNs to solve the "cold start" location prediction problem, which can be analog to the location recommendation problem specifically on a user's next check-in. In [37, the authors utilized both user-based and friend-based collaborative filtering for location recommendation. This approach did not consider the geographical property of social correlations, and could be related to the equal strength and single measure version of our gSCorr. Cho et al. 9] studied the periodic patterns of check-in behavior on LBSNs, and proposed a Gaussian mixture model together with the social network information considered for location prediction, while their results also show limited improvements from social network. Gao et al. [15] studied the social-historical ties on Foursquare, and found that both ties have contributions to the user's check-in behavior, while social ties are complementary to the historical ties, especially when the historical model does not perform well due to the long and noisy history.

\section{Conclusions and Future Work}

In this paper, we propose a geo-social correlation model to capture the social correlations of check-in behavior on LBSNs. We investigate the correlations in context of social networks and geographical distance. The work presented in this paper suggests many future directions. Firstly, the geographical separation of social relationships in this work is binary. It would be interesting to consider a continuous function of social correlations with the changing of geographical distance. Secondly, in this work we focus on utilizing social network information to solve the cold-start problem, while ignoring a user's own check-in history. In the future we will continue to study how to take advantage of both social correlations and historical check-ins, and explore novel usage of such information. 
Acknowledgements This work is supported, in part, by ONR (N000141010091).

\section{References}

1. Anagnostopoulos, A., Kumar, R., Mahdian, M.: Influence and correlation in social networks. In: KDD, pp. 7-15. ACM (2008)

2. Anderson, J., Michalski, R., Michalski, R., Carbonell, J., Mitchell, T.: Machine learning: An artificial intelligence approach. Morgan Kaufmann (1986)

3. Backstrom, L., Sun, E., Marlow, C.: Find me if you can: improving geographical prediction with social and spatial proximity. In: Proceedings of the 19th international conference on World wide web, pp. 61-70. ACM (2010)

4. Barnes, S., Scornavacca, E.: Mobile marketing: the role of permission and acceptance. International Journal of Mobile Communications 2(2), 128-139 (2004)

5. Boyd, S.P., Vandenberghe, L.: Convex optimization. Cambridge university press (2004)

6. Cairncross, F.: The death of distance: How the communications revolution is changing our lives. Harvard Business Press (2001)

7. Chang, J., Sun, E.: Location 3: How users share and respond to location-based data on social networking sites. ICWSM (2011)

8. Cheng, Z., Caverlee, J., Lee, K., Sui, D.: Exploring millions of footprints in location sharing services. In: ICWSM (2011)

9. Cho, E., Myers, S., Leskovec, J.: Friendship and mobility: user movement in locationbased social networks. In: KDD, pp. 1082-1090. ACM (2011)

10. Easley, D., Kleinberg, J.: Networks, crowds, and markets. Cambridge Univ Press (2010)

11. Gao, H., Barbier, G., Goolsby, R.: Harnessing the crowdsourcing power of social media for disaster relief. Intelligent Systems, IEEE 26(3), 10-14 (2011)

12. Gao, H., Huan: Data analysis on location-based social netwoks. In: A. Chin, D. Zhang (eds.) Mobile Social Networking: An Innovative Approach, pp. 165-194. Springer (2013)

13. Gao, H., Tang, J., Hu, X., Liu, H.: Exploring temporal effects for location recommendation on location-based social networks. In: Proceedings of the 7th ACM conference on Recommender systems, pp. 93-100. ACM (2013)

14. Gao, H., Tang, J., Hu, X., Liu, H.: Modeling temporal effects of human mobile behavior on location-based social networks. In: Proceedings of the 22nd ACM international conference on Conference on information \& knowledge management, pp. 1673-1678. ACM (2013)

15. Gao, H., Tang, J., Liu, H.: Exploring social-historical ties on location-based social networks. In: ICWSM (2012)

16. Gao, H., Tang, J., Liu, H.: gscorr: Modeling geo-social correlations for new check-ins on location-based social networks. the 21st ACM International Conference on Information and Knowledge Management (2012)

17. Gao, H., Tang, J., Liu, H.: Mobile location prediction in spatio-temporal context. Nokia Mobile Data Challenge Workshop (2012)

18. Goldenberg, J., Levy, M.: Distance is not dead: Social interaction and geographical distance in the internet era. Arxiv preprint arXiv:0906.3202 (2009)

19. Goodchild, M., Glennon, J.: Crowdsourcing geographic information for disaster response: a research frontier. International Journal of Digital Earth 3(3), 231-241 (2010)

20. Kessler, S.: Foursquare tops 20 million users. http://mashable.com/2012/04/16/foursquare20-million/ (2012)

21. Li, S.: Location based services marketing. Master thesis, Royal Institute of Technology (2011)

22. Long, X., Jin, L., Joshi, J.: Exploring trajectory-driven local geographic topics in foursquare. In: UbiComp, pp. 927-934 (2012)

23. Long, X., Joshi, J.: A hits-based poi recommendation algorithm for location-based social networks. In: 2013 IEEE/ACM International Conference on Advances in Social Networks Analysis and Mining (2013)

24. Mok, D., Wellman, B., Carrasco, J.: Does distance matter in the age of the internet? Urban Studies 47(13), 2747 (2010) 
25. Monreale, A., Pinelli, F., Trasarti, R., Giannotti, F.: Wherenext: a location predictor on trajectory pattern mining. In: Proceedings of the 15th ACM SIGKDD international conference on Knowledge discovery and data mining, pp. 637-646. ACM (2009)

26. Nocedal, J., Wright, S.: Numerical optimization. Springer verlag (1999)

27. Noulas, A., Scellato, S., Mascolo, C., Pontil, M.: An empirical study of geographic user activity patterns in foursquare. ICWSM (2011)

28. Scellato, S., Mascolo, C., Musolesi, M., Latora, V.: Distance matters: Geo-social metrics for online social networks. In: Proceedings of the 3rd conference on online social networks, pp. 8-8. USENIX Association (2010)

29. Scellato, S., Musolesi, M., Mascolo, C., Latora, V., Campbell, A.: Nextplace: a spatiotemporal prediction framework for pervasive systems. Pervasive Computing pp. 152-169 (2011)

30. Scellato, S., Noulas, A., Lambiotte, R., Mascolo, C.: Socio-spatial properties of online location-based social networks. ICWSM 11 (2011)

31. Scellato, S., Noulas, A., Mascolo, C.: Exploiting place features in link prediction on location-based social networks. In: Proceedings of the 17th ACM SIGKDD international conference on knowledge discovery and data mining, pp. 1046-1054. ACM (2011)

32. Spaccapietra, S., Parent, C., Damiani, M., De Macedo, J., Porto, F., Vangenot, C.: A conceptual view on trajectories. Data and knowledge engineering 65(1), 126-146 (2008)

33. Su, X., Khoshgoftaar, T.M.: A survey of collaborative filtering techniques. Advances in Artificial Intelligence 2009, 4 (2009)

34. Thanh, N., Phuong, T.: A gaussian mixture model for mobile location prediction. In: 2007 IEEE International Conference on Research, Innovation and Vision for the Future, pp. 152-157. IEEE (2007)

35. Wang, X., Tang, L., Gao, H., Liu, H.: Discovering overlapping groups in social media. In: Data Mining (ICDM), 2010 IEEE 10th International Conference on, pp. 569-578. IEEE (2010)

36. Ye, M., Yin, P., Lee, W.: Location recommendation for location-based social networks. In: Proceedings of the 18th SIGSPATIAL International Conference on Advances in Geographic Information Systems, pp. 458-461. ACM (2010)

37. Ye, M., Yin, P., Lee, W., Lee, D.: Exploiting geographical influence for collaborative point-of-interest recommendation. In: Annual International ACM SIGIR Conference on Research and Development in Information Retrieval, pp. 325-334 (2011)

38. Zickuhr, K., Smith, A.: $28 \%$ of american adults use mobile and social location-based services. Pew Internet and American Life Center Report. Retrieved September 8, 2011 (2011) 